\section{Use of anomalous small-angle $X$-ray scattering for the investigation of highly charged colloids}

\author{
N. Dingenouts, ${ }^{a}$ R. Merkle, ${ }^{a}$ X. Guo, ${ }^{a}{ }^{T}$. Narayanan, ${ }^{b}$ \\ G. Goerigk ${ }^{\mathrm{c}}$ and M. Ballauff ${ }^{\mathrm{a}} *$ \\ ${ }^{a}$ Polymer-Institut, Universität Karlsruhe, Kaiserstrasse 12, D-76128 \\ Karlsruhe, Germany, ${ }^{b}$ ESRF, B.P. 220, 3843 Grenoble Cedex, France, ${ }^{c}$ Institut für \\ Festkörperforschung, Forschungszentrum Jülich, Postfach 1913, 52425 Jülich, \\ Germany. E-mail: Matthias.Ballauff@chemie.uni-karlsruhe.de
}

We present a study of the radial structure of a spherical polyelectrolyte brushes by anomalous small-angle X-ray scattering. The spherical polyelectrolyte brushes consist of a solid poly(styrene) core of $c a$. $100 \mathrm{~nm}$ diameter onto which long linear chains of poly(acrylic acid) (PAA) are densely grafted. A sufficiently high $\mathrm{pH}$, these polyelectrolyte chains are fully charged. Rubidium ions are used as counterions because their adsorption edge $(15199.6 \mathrm{eV})$ can be conventiently reached by synchrotron radiation. By performing small-angle X-ray scattering studies at different energies of the incident radiation, the contribution of the $\mathrm{Rb}^{+}$counterions can be analyzed separately. The scattering contribution of the counterions can be derived and compared to the scattering intensity of the entire particle. The distributions of the macro-ion and of the counterions are shown to be very similiar. This shows that the counterions must be confined within the brush.

Keywords: ASAXS; counterion condensation; latex; particles; polyelectrolytes.

\section{Introduction}

Polyelectrolytes consist of a highly charged macro-ion and its respective counterions. When dispersed in media of sufficient dielectric constant as e.g. water the counterions dissociate from the macro-ion (Mandel, 1987). The distribution of the counterions in the strong electric field of the macro-ion is among the classical questions in this field. Small-angle X-ray scattering (SAXS) is well-suited to study this problem experimentally if the counterions exhibit enough contrast towards the solvent (Wu et al., 1988). Often the contribution of the macro-ion is non-negligibe which renders the determination of the structure of the clouds of counterions less secure.

Anomalous small-angle X-ray scattering (ASAXS) can solve this problem if the adsorption edge of the counterions can be reached experimentally (Stuhrmann et al., 1991). Hence, changing the energy of the incident beam changes the scattering factor of the counterions while leaving the scattering contribution of the macro-ion constant. Recently, we have demonstrated that ASAXS gives valuable information on dissolved polyelectrolytes that cannot be gained by SAXS or other methods (Guilleaume et al., 2001, Guilleaume et al., 2002). Model calculations showed that ASAXS should be of similar use in the case of spherical macro-ions (de Robillard et al., 2001).

In this communication we present the first analysis of a well-defined polymeric macro-ion by ASAXS. The system investigated here consists of a dilute aqueous solution of the spherical polyelectrolyte brushes (SPB) depicted schematically in Fig. 1. The particles consists of a solid poly(styrene) core onto which linear chains of poly(acrylic acid) (PAA) are attached. The PAA chains affixed to the surface are fully dissociated and the negative charge of each group is balanced by a $\mathrm{Rb}^{+}$counterion. The polyelectrolyte chains are rather dense and their mutual distance on the surface of the core particles is small (brush limit). These systems have recently been studied comprehensively

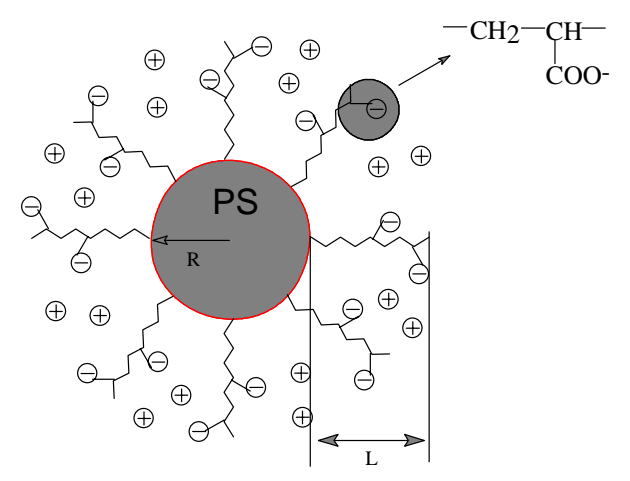

Figure 1

Scheme of the spherical polyelectrolyte brushes (SPB) analyzed in this study. The particles consist of a poly(styrene) core of ca. $100 \mathrm{~nm}$ diameter. Onto this core linear poly(acrylic acid) (PAA) chains are grafted chemically. The distance between the chains on the surface of the core particle is much smaller than the linear dimensions of the PAA chains. Hence, the PAA chains form a brush in which the $\mathrm{Rb}^{+}$counterions are confined.

by dynamic light scattering (Guo \& Ballauff, 2000; Guo \& Ballauff, 2001). Here we demonstrate that these particles are ideally suited for an ASAXS-study. The energy of the incident radiation was changed to reach the adsorption edge of Rubidium (15 $199.6 \mathrm{eV}$; see Fig. 2) from below. Near the adsorption edge the scattering length of the counterion becomes a complex function. The macro-ion is made up from polymers that exhibit no anomalous dispersion in the energy range used here. Hence, ASAXS can be used to extract the scattering contribution of the counterions as will be shown below.

\section{Theory}

\subsection{Small-angle scattering from spherical polyelectrolyte brushes}

The absolute scattering intensity $I(q)$ is given by

$$
I(q)=\frac{N}{V} I_{0}(q) S(q)
$$

where $N / V$ is the number of dissolved particles per volume, $I_{0}(q)$ is the scattering intensity of a single particle, $q=4 \pi \sin \theta / \lambda$ is the modulus of the scattering vector, with $\theta=$ half the scattering angle and $\lambda$ = wavelength of the incident X-rays, and $S(q)$ is the structure factor. Since we work in the dilute regime, the influence of $S(q)$ is restricted to the region of smallest angles (Ballauff, 2001). Hence, in the following $S(q)=1$ which corresponds to a system of non-interacting particles. Despite the spherical symmetry the scattering intensity $I_{0}(q)$ is a complex quantity because the scattering factor of the $\mathrm{Rb}^{+}$ions has a nonvanishing imaginary part.Therefore

$$
I_{0}(q)=F(q) F^{*}(q)
$$


where $F(q)$ is the scattering amplitude of a single particle. Because of the spherical symmetry of the particles $F(q)$ follows as

$$
F(q)=4 \pi \int_{0}^{\infty}\left[\rho(r)-\rho_{\mathrm{m}}\right] \frac{\sin q r}{q r} r^{2} \mathrm{~d} r
$$

where $\rho(r)$ is the radial electron density of the spherical polyelectrolyte brush and $\rho_{\mathrm{m}}$ is the electron density of the surrounding medium water.

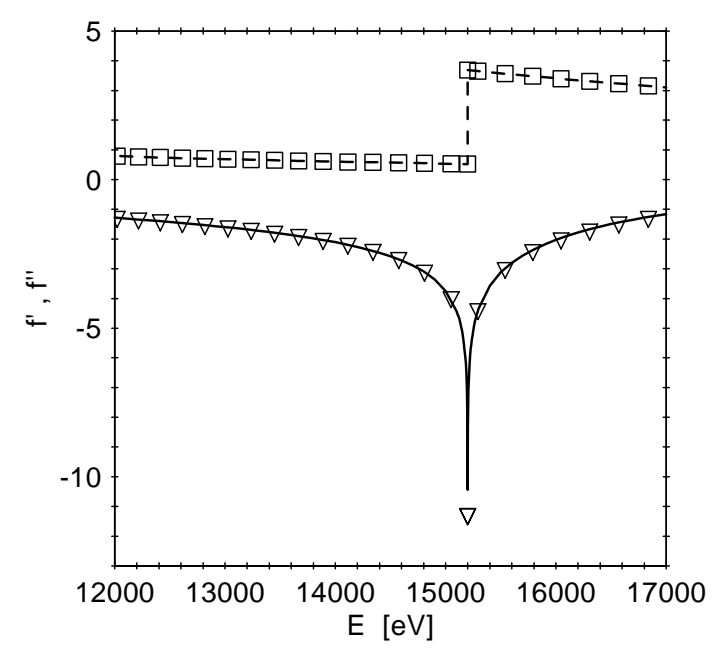

Figure 2

Scattering factor of Rubidium near the K-edge: the real and imaginary parts $f^{\prime}$ and $f^{\prime \prime}$ as function of the energy $E$ of the incident radiation. Triangle: $f^{\prime}$ taken from the tabulation of Henke et al. (1993); solid line: $f^{\prime}$ taken from the calculation of Brennan and Cowan (1992) squares connected by dashed lines: $f^{\prime \prime}$ taken from the tabulation of Henke et al. (1993).

The excess electron density $\rho(r)-\rho_{\mathrm{m}}$ consists of two parts: i) The contribution of the macro-ion, and ii) the contribution of the counterions which is complex.

\subsection{Contrast of macro-ion:}

The macro-ion is composed of a poly(styrene) core having an excess electron density $\Delta \rho_{\text {core. }}$ This contrast is small and the main contributions stem from the PAA chains attached to the surface. With the radial excess electron density of the PAA chains given by $\Delta \rho_{\mathrm{PAA}}(r)$ we have for the radial excess electron density of the macro-ion

$$
\begin{array}{r}
\Delta \rho_{\text {macroion }}(r)=\Delta \rho_{\text {core }} ; r \leq R_{\mathrm{c}} \\
\Delta \rho_{\text {macroion }}(r)=\Delta \rho_{\text {PAA }}(r) ; r>R_{\mathrm{c}}
\end{array}
$$

where $R_{\mathrm{c}}$ is the radius of the core.

\subsection{Complex contrast of counterions:}

The scattering factor $f_{\text {ion }}$ becomes a complex function of the energy $E$ of the incident radiation near the adsorption edge of the ions:

$$
f_{\text {ion }}=f_{0}+f^{\prime}(E)+\tilde{i} f^{\prime \prime}(E)
$$

The first term $f_{0}$ is the non-resonant term which equals the atomic number of the element. The second and the third term in equation (5) are the real and the imaginary part due to the anomalous dispersion near the adsorption edge and $\tilde{i}$ is the complex unit. The imaginary part $f^{\prime \prime}(E)$ is directly related to the adsorption cross section for X-rays of energy $E$. Both $f^{\prime}(E)$ and $f^{\prime \prime}(E)$ are related to each other by the Kramers-Kronig relation. Fig. 2 displays the variation of $f^{\prime}(E)$ and of $f^{\prime \prime}(E)$ calculated for Rubidium from the tabulation of Henke et al. (1993) and from the calculations of Brennan and Cowan (1992). Both sets of data agree well and provide a sound base for the evaluation of the present data.

It is interesting to note that the imaginary part $f^{\prime \prime}$ remains practically constant below the edge whereas $f^{\prime}$ shows a strong variation near the edge. Hence, the ASAXS effect will be fully determined by the real part $f^{\prime}$ if the experiments are conducted at energies below the adsorption edge. Measurements above the edge, on the other hand, would lead to strong fluorescence which may mask the scattering intensity at high $q$. Fig. 2 also demonstrates that measurements need to be done very closely to the adsorption edge if a sufficient decrease of $f^{\prime}$ is to be observed.

For ions immersed in a medium with electron density $\rho_{\mathrm{m}}$ the number of excess electrons per ion $\Delta f_{\text {ion }}$ follows as

$$
\begin{array}{r}
\Delta f_{\text {ion }}=f_{0}-\rho_{\mathrm{m}} V_{\text {ion }}+f^{\prime}(E)+\tilde{i} f^{\prime \prime}(E)= \\
\Delta f_{0}+f^{\prime}(E)+\tilde{i} f^{\prime \prime}(E)
\end{array}
$$

where $V_{\text {ion }}$ is the volume of a single ion. The quantity $\Delta f_{0}$ hence denotes the scattering contribution of a single counterion that is independent of the energy of the incident radiation.

Let $n_{\text {ion }}(r)$ be the number of counterions per unit volume within distance $r$ from the center of the particle. Then the contribution of the counterions to the excess electron density of the entire particles is given by

$$
\Delta \rho_{\text {ion }}(r)=n_{\text {ion }}(r) \Delta f_{\text {ion }} ; r \geq R_{\mathrm{c}}
$$

From these considerations it follows that the excess electron density of the spherical polyelectrolyte brush is given by

$$
\begin{array}{r}
\Delta \rho(r)=\Delta \rho_{\text {core }}(r) ; r \leq R_{\mathrm{c}} \\
\Delta \rho(r)=\Delta \rho_{\text {PAA }}(r)+\Delta f_{\text {ion }} ; r>R_{\mathrm{c}}
\end{array}
$$

\subsection{Calculation of $I_{0}(q)$ and of $v(q)$ :}

The scattering amplitude $F(q)$ is split into a non-resonant term $F_{0}(q)$ and an energy-dependent term $F_{\text {res }}(q)$ :

$$
F(q)=F_{0}(q)+F_{\text {res }}(q)
$$

For the non-resonant term we obtain from equation (3)

$$
\begin{aligned}
F_{0}(q)= & 4 \pi \int_{0}^{\infty} \Delta \rho_{\text {macroion }} \frac{\sin q r}{q r} r^{2} \mathrm{~d} r+ \\
& +\Delta f_{0} 4 \pi \int_{R_{\mathrm{c}}}^{\infty} n_{\text {ion }}(r) \frac{\sin q r}{q r} r^{2} \mathrm{~d} r
\end{aligned}
$$

The resonant term follows as

$$
\begin{aligned}
F_{\text {res }}(q)=\left(f^{\prime}(E)+\tilde{i} f^{\prime \prime}(E)\right) & 4 \pi \int_{R_{\mathrm{c}}}^{\infty} n_{\text {ion }}(r) \frac{\sin q r}{q r} r^{2} \mathrm{~d} r \\
& =\left(f^{\prime}(E)+\tilde{i} f^{\prime \prime}(E)\right) v(q)
\end{aligned}
$$

where $v(q)$ is the Fourier-transform of the distribution function of the counterions $n_{\text {ion }}(r)$. Equation (2) then leads to

$$
I_{0}(q)=F_{0}^{2}(q)+2 f^{\prime} F_{0}(q) v(q)+\left(f^{\prime}(E)^{2}+f^{\prime \prime}(E)^{2} v^{2}(q)\right.
$$


Equation (12) shows that the intensity measured near the adsorption edge consists of three parts: The term $F_{0}^{2}(q)$ denote the non-resonant intensity that is measured far from the edge by the conventional SAXS experiment. The second term is the cross term of the non-resonant and the resonant amplitude of the object. It scales linearly with $f^{\prime}$ and presents in most cases the leading part of the ASAXS effect. It should be kept in mind that the amplitude $F_{0}(q)$ embodied in this term is still related to the entire scattering object, i.e.,

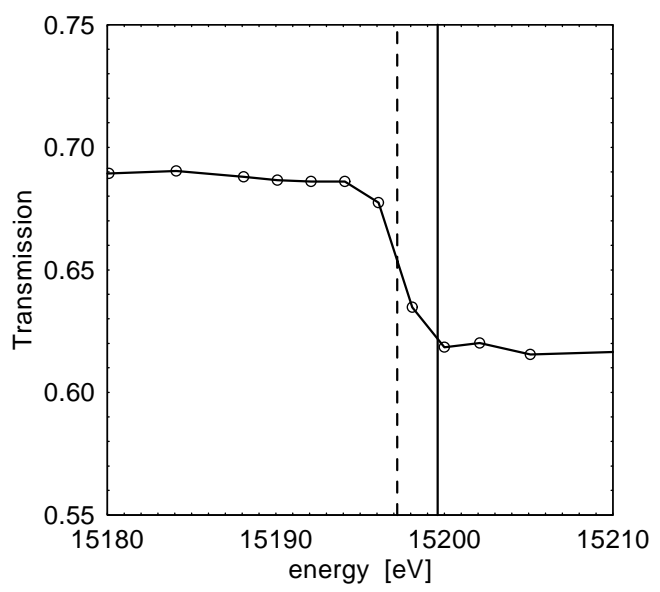

\section{Figure 3}

Determination of the position of the adsorption edge. The transmission of a solution of the spherical polyelectrolyte brush is plotted against the energy of the incident beam. The edge determined from the point of inflection of the experimental transmission curve is slightly shifted from the theoretical value (solid line). This shift is caused by a residual uncertainty of the adjustment of the monochromator. It is corrected for in all subsequent calculations.

to the spatial distribution of the non-resonant scatteres as well. Only the third term is solely related to the spatial distribution of resonantly scattering parts of the object under consideration. Its prefactor is much smaller, however, and its magnitude becomes only appreciable if the second term in equation (12) is small. This is borne out directly from the model calculations presented by de Robillard et al. (2001).

For systems having $F_{0}(q)$ of appreciable magnitude, the third term in equation (12) can be omitted and $v(q)$ can be directly calculated from the second term of equation (12). Hence, $v(q)$ is obtained by dividing the experimental cross term $F_{0}(q) v(q)$ through the amplitude $F_{0}(q)$ that is available from the first term of equation (12), i.e, from the conventional SAXS experiment. Since we are dealing with spherosymmetric systems, $F_{0}(q)$ may directly obtained from the SAXS intensity. This procedure leads to $v(q)$ which may directly be inverted to yield the radial distribution $n_{\text {ion }}(r)$ of the counterions. It must be kept in mind, however, that the spherical polyelectrolyte brushes under consideration here are always slightly polydisperse. The finite width of the size distribution of the particles will smear out the minima at higher scattering angles and the analysis of the ASAXS data directly for $v(q)$ must take this effect into account. This can be done by defining a "measured amplitude" $v_{\mathrm{M}}(q)$ from equation (12) through

$$
v_{\mathrm{M}}(q)=\frac{\sum_{i} F_{0, i}(q) v_{i}(q)}{\operatorname{sign}\left[F_{0}(q)\right]\left[\sum_{i} F_{0, i}^{2}(q)\right]^{1 / 2}}
$$

If the polydispersity is small, $v_{\mathrm{M}}(q)$ leads directly to $n_{\text {ion }}(r)$. In case of a broader size distribution, $v_{\mathrm{M}}(q)$ may be compared to theoretical models and the radial distribution of the counterions follows from a fit procedure.

\section{Experimental}

\subsection{Materials and methods}

The particles used in this study is the system L22 (Guo \& Ballauff, 2001). The latex was purified by extensive ultrafiltration to remove the

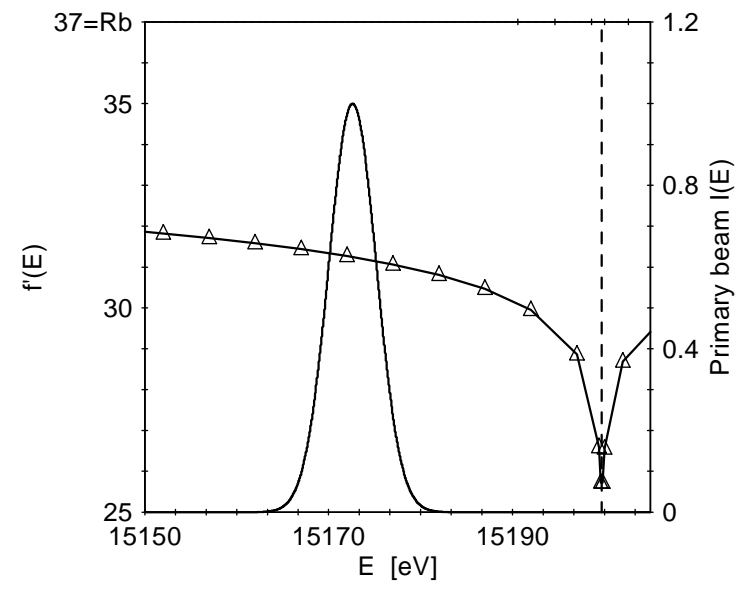

Figure 4

Influence of the energy dispersion of the primary beam. The energy spread of the primary beam is compared to the dependence of $f^{\prime}$ on the energy of the incident beam. The effective $f_{\text {eff }}^{\prime}$ results from the convolution of the energy spread with $f^{\prime}(E)$.

free polymer in the serum. The characterization with regard to the contourlength $L_{\mathrm{c}}$ and the grafting density was done as described recently (Guo \& Ballauff, 2000). The particles studied here had a core diameter of $68 \mathrm{~nm}$, a weight-average contourlength $L_{\mathrm{c}}=233 \mathrm{~nm}$ and a grafting density $\sigma=0.026 \mathrm{~nm}^{-2}$ (cf. Table II of (Guo \& Ballauff, 2001)).

The $\mathrm{Rb}^{+}$counterions were introduced by titration of the acidic particles by aqueous $\mathrm{RbOH}$. Previous studies have shown that all carboxylgroups within the brush are converted by this procedure (Guo \& Ballauff, 2001). No additional salt was added to the system studied here. $\mathrm{RbOH}$ was added until a $\mathrm{pH}$ of 10 was reached. This ensured full ionization of the brushes.

All ASAXS experiments reported here were performed using the ID2-beamline of the ESRF in Grenoble. The sample-to-detector distance was $10 \mathrm{~m}$ in all cases. As detector an image-intensified CCDcamera was used. The energy of the incident beam was varied between $12.460 \mathrm{eV}$ and $15200 \mathrm{eV}$. All data have been corrected for detector response and normalized to an absolute scale. The normalized data were azimutally averaged and corrected for the background of the solvent water and the empty cell. The possible contribution from fluorescence that occurs was checked by measurements at energies slightly above the edge. It turned out that data taken in the $q$-range under consideration here is not influenced by fluorescence.

The particle concentration was $6 \mathrm{wt} . \%$ to minimize the effect of particle interaction. Previous investigation had demonstrated that this concencentration is low enough to disregard the influence of mutual interaction of the particles in the $q$-range analyzed here (see Ballauff, 2001, and further citations given there).

Special care has been taken to localize exactly the energy of the edge by careful measurements of the absorption of each sample. Fig. 3 displays 
as an typical exampe the transmission of a given solution as function of the energy of the incident beam.

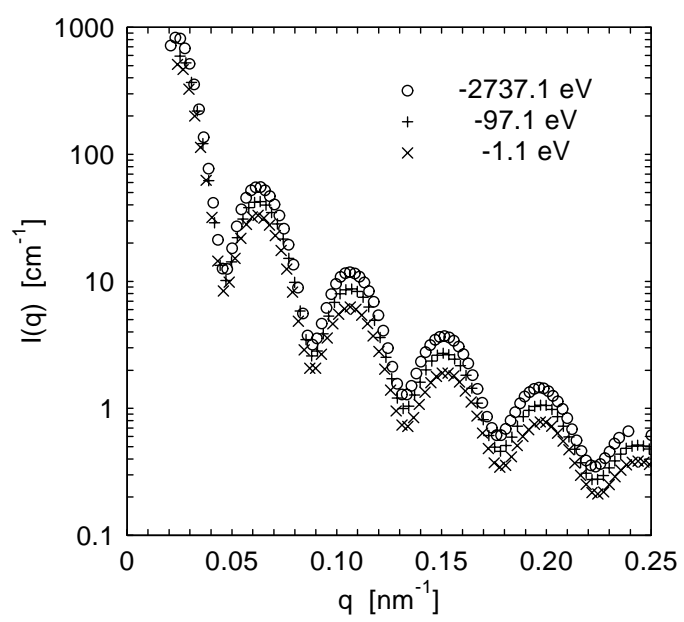

Figure 5

Experimental scattering intensities measured at different energies of the incident beam.

The solid line marks the theoretical value of the energy of the edge $(15199.6 \mathrm{eV})$ whereas the broken line shows the point of inflection of the absorption curve. This shows that there is a small shift of the energy. This small shift was taken into account for the determination of the true energy of the incident beam.

The ASAXS experiment of a given solution was done as follows: First the energy scale of the monochromator was corrected for the shift seen in the transmission curve. Then intensities are measured at energies below the edge thus determined. All scattering curve are corrected for the sensitivity of the detector using software available at the beamline.

An important point to be considered in the course of an ASAXS experiments is the energy spread of the primary beam. For the ID2 $\Delta E / E=2 \times 10^{-4}$. Fig. 4 shows the real part $f^{\prime}$ together with the profile of the primary beam. The narrow width renders the effect of a finite width of the primary beam negligible except for the immediate neighborhood of the edge $(\Delta E<10 \mathrm{eV})$. For this region an effective scattering factor $f_{\text {eff }}^{\prime}$ has been calculated by weighting $f^{\prime}(E)$ by the profile of the primary beam the maximum of which was shifted to the respective energy $E$. Table 1 lists the energies at which measurements have been conducted together with the respective $f^{\prime}(E)$ and $f_{\text {eff }}^{\prime}(E)$. In all subsequent calculations $f_{\mathrm{eff}}^{\prime}(E)$ was used.

\section{Table 1}

Scattering factors $f^{\prime}$ and $f_{\text {eff }}^{\prime}$ as the function of the distance $\Delta E$ to the adsaorption edge.

\begin{tabular}{lll}
\hline$\Delta E[\mathrm{eV}]^{\dagger}$ & $f^{\prime \ddagger}$ & $f_{\text {eff }}^{\prime} \S$ \\
\hline-2737 & -1.41 & -1.41 \\
-397.1 & -3.09 & -3.00 \\
-197.1 & -3.76 & -3.71 \\
-97.1 & -4.46 & -4.45 \\
-13.1 & -6.51 & -6.33 \\
-1.1 & -9.65 & -8.77 \\
\hline
\end{tabular}

$\dagger$ Difference of energy relative to the energy of the edge

$\ddagger$ Real part of the scattering factor (Henke et al., 1993)

$\S$ Effective scattering factor used in the evaluation of the data; see the discussion of Fig. 4

\section{Results and discussion}

All ASAXS experiments discussed here are done using spherical polyelectrolyte brushes L22 synthesized and characterized recently (Guo $\&$ Ballauff, 2001). For a pH of 10 used here these particles are fully charged. Fig. 5 displays the intensities of this suspension measured at different energies of the incident beam. For the sake of clarity only three scattering curves are shown. The circles refer to an energy far below the edge where $f^{\prime}$ and $f^{\prime \prime}$ are very small. First of all, the distinct maxima and minima of the scattering curves are clearly seen and point to the narrow size distribution of the spherical polyelectrolyte brushes studied here. Moreover, it is seen that the measured intensities become smaller upon approaching the adsorption edge. This becomes directly evident upon inspection of equation (12). As demonstrated by the model calculation discussed in de Robillard et al. (2001) the third term in equation (12) is small and the ASAXS effect is dominated by cross amplitude which is negative by its prefactor $f^{\prime}$ (see Fig. 2).

The magnitude of $f^{\prime}$ is increasing when approaching the energy of the adsorption edge and the measured intensity is progressively diminished by the negative ASAXS contribution. Equation (12) suggests to remove the non-resonant part of the intensity by subtracting $I_{0}(q)$ measured far below the adsorption edge.

The resulting plot is displayed in Fig. 6. The solid line shows the intensity measured far below the edge whereas the other data refer to the differences of this intensity and the intensities measured near the edge. Evidently, the cross amplitude (see equation (12)) is of appreciable magnitude near the adsorption edge. Accurate data can be taken, however, even $400 \mathrm{eV}$ below the edge. Hence, the ASAXS effect is conveniently measurable for the present systems.

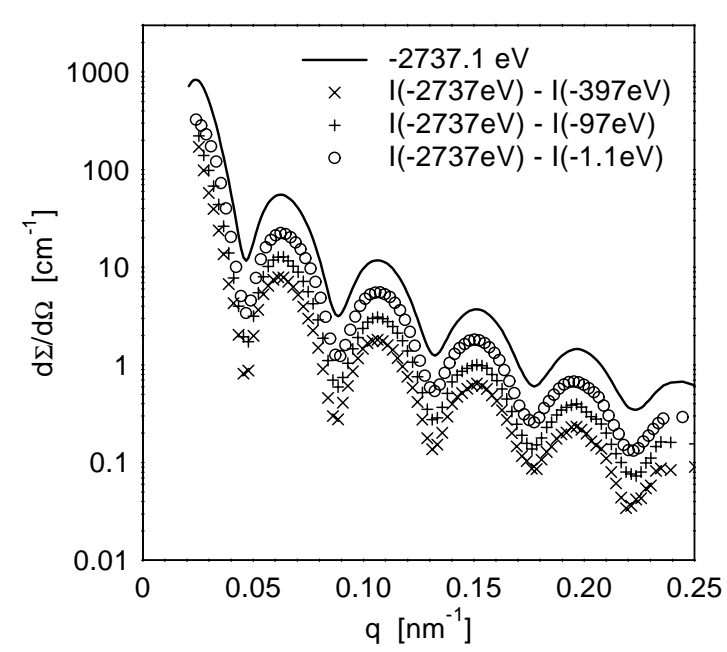

Figure 6

Difference of experimental scattering intensities measured at different energy of the incident beam.

A feature comanding attention is the parallel shift of the difference curves. The maxima remain at the same $q$-values and the different energies lead only to a shift parallel to the ordinate. A similar effect has already been seen in the model calculations discussed recently (de Robillard et al., 2001). They point to the fact that the cross term of equation (12) is the leading part of the non-resonant terms. This becomes obvious when normalizing the difference curves displayed 
in Fig. 5 to $f_{\text {eff }}^{\prime}$. Fig. 7 shows the resulting data refering to different energies of the incident beam. All difference curves are now located on a single master curve that corresponds to $F_{0}(q) v(q)$. This result gives the experimental proof that the cross term is much larger than the third term in equation (12) which is solely due to resonant terms $f^{\prime}$ and $f^{\prime \prime}$.

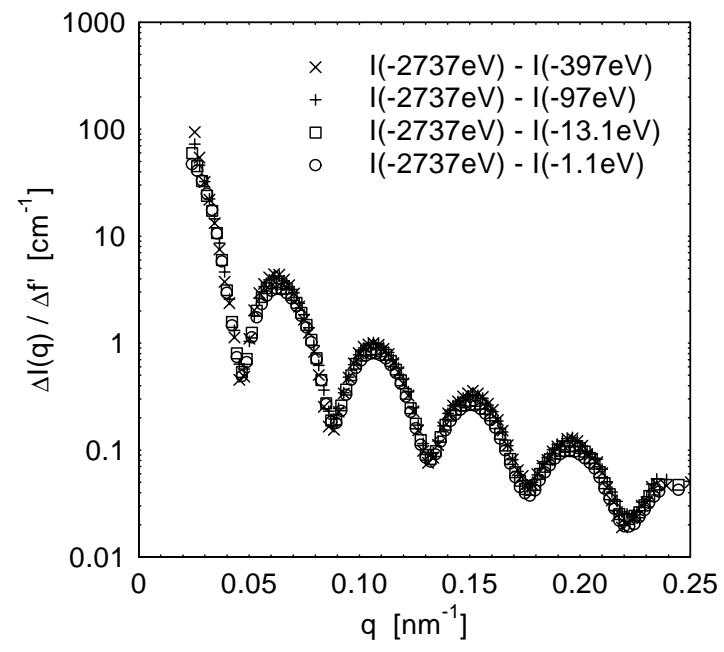

Figure 7

Difference of experimental scattering intensities measured at different energy of the incident beam normalized to $f_{\text {eff }}^{\prime}$.

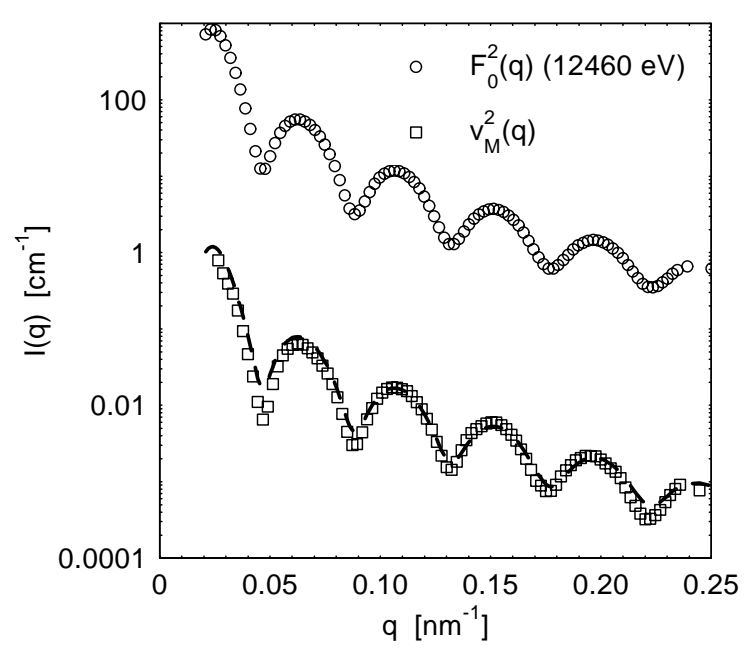

Figure 8

The square of the measured amplitude $v_{\mathrm{M}}(q)$ (see equation (13)) in comparison to the conventional SAXS intensity $F_{0}(q)^{2}$.
The master curve displayed in Fig. 7 is the central experimental result of the present analysis. It shows that ASAXS does not allow to see directly the contribution $v(q)$ of the $\mathrm{Rb}^{+}$ions to the measured intensity which is embodied in the third term of equation (12). The contrast of the macro-ion which includes the high X-ray contrast of the attached PAA chains is high enough to render the amplitude $F_{0}(q)$ a non-negligible quantity. The cross term $F_{0}(q) v(q)$, however, is linear independent of the non-resonant term $F_{0}(q)$ and thus present highly valuable information on the Fourier-transform $v(q)$ of the distribution $n_{\text {ion }}(r)$.

Moreover, as already discussed above, ASAXS allows to determine directly the "measured scattering amplitude" $v_{\mathrm{M}}(q)$ of the counterion cloud by equation (13). Evidently, $v_{\mathrm{M}}^{2}(q)$ is then the scattering intensity of the cloud of counterions around the macro-ion. Fig. 8 shows $v_{\mathrm{M}}^{2}(q)$ together with $F_{0}^{2}(q)$. Fig. 8 demonstrates that the information solely due to the distribution of counterions can be obtained by a carefully conducted experiment. Moreover, Fig. 8 shows that this information can be obtained with excellent accuracy.

A comparison of $v^{2}(q)$ with suitable models gives the distribution $n_{\text {ion }}(r)$. The $q$-range available here, however, is to small to arrive at meaningful results. Therefore an extension of the present measurements to higher scattering angles is necessary. The results obtained so far, however, already indicate that the radial distribution of the counterions must be closely related to the radial structure of the macro-ion. This can directly be argued from the fact that $v_{\mathrm{M}}^{2}(q)$ and $F_{0}^{2}(q)$ are related in good approximation by a factor. This is directly demonstrated in Fig. 8: The dashed line is the intensity $F_{0}^{2}(q)$ shifted parallel to the ordinate to match the intensity $v_{\mathrm{M}}^{2}(q)$. Full agreement is seen which shows that $v_{\mathrm{M}}^{2}(q)$ differs from $F_{0}^{2}(q)$ only by a constant factor related to the different contrast of the entire system and the counterions alone. This results therefore clearly demonstrates that the counterions merely decorate the polyelectrolyte chains of the macro-ion.

Acknowledgements Financial support by the Deutsche Forschungsgemeinschaft, Schwerpunkt "Polyelektrolyte", and by the European Community, Project "HUSC", is gratefully acknowledged. We acknowledge allocation of beamtime by the European Synchrotron Radiation Facility in Grenoble (SC-787).

\section{References}

Ballauff, M. (2001). Curr. Opinion Colloid Interf. Sci. 6, 132-139. Brennan, S. \& Cowan, P. L. (1992). Rev. Sci. Instrum. 63, 850-853.

Guo, X. \& Ballauff, M. (2000). Langmuir 16, 8719-8726.

Guo, X. \& Ballauff, M. (2001). Phys. Rev. E 64, 051406/1-051406/9.

Guilleaume, B., Ballauff, M., Goerigk, G., Wittemann, M. \& Rehahn, M. (2001). Colloid Polym. Sci. 279, 829-835.

Guilleaume, Blaul, J., B., Ballauff, M., Wittemann, M., Rehahn, M. \& Goerigk, G., (2002). Eur. Phys. J. E 8, 299-309.

Henke, B. L., Gullikson, E.M. \& Davis, J. C. (1993). Atomic Data and Nuclear Data Table, 54, 181-342.

de Robillard, Q., Guo, X. \& Ballauff, M. (2001). Macromol. Symp. 164, 81-90.

Stuhrmann, H. B., Goerigk, G., \& Munk, B. (1991). Handbook of Synchrotron Radiation Vol. 4, chapter 17, edited by Ebashi, S., Koch, M. \& Rubenstein, E. Amsterdam: Elsevier.

Wu, C. F., Chen, S.H., Shih, L. B. \& Lin, S. H. (1988). Phys. Rev. Lett. 61, 645-648. 